\title{
An improved Hardy type inequality on Heisenberg group
}

Ying-Xiong Xiao

Correspondence: yxxiao2011@163. com

School of Mathematics and Statistics, Xiaogan University, Xiaogan, Hubei, 432000, People's Republic of China

\section{Abstract}

Motivated by the work of Ghoussoub and Moradifam, we prove some improved Hardy inequalities on the Heisenberg group $\mathbb{H}^{n}$ via Bessel function.

Mathematics Subject Classification (2000):

Primary 26D10

Keywords: Hardy inequality, Heisenberg group

\section{Introduction}

Hardy inequality in $\mathbb{R}^{N}$ reads, for all $u \in C_{0}^{\infty}\left(\mathbb{R}^{N}\right)$ and $N \geq 3$,

$$
\int_{\mathbb{R}^{N}}|\nabla u|^{2} \mathrm{~d} x \geq \frac{(N-2)^{2}}{4} \int_{\mathbb{R}^{N}} \frac{u^{2}}{|x|^{2}} \mathrm{~d} x
$$

and $\frac{(N-2)^{2}}{4}$ is the best constant in (1.1) and is never achieved. A similar inequality with the same best constant holds in $\mathbb{R}^{N}$ is replaced by an arbitrary domain $\Omega \subset \mathbb{R}^{N}$ and $\Omega$ contains the origin. Moreover, in case $\Omega \subset \mathbb{R}^{N}$ is a bounded domain, Brezis and Vázquez [1] have improved it by establishing that for $u \in C_{0}^{\infty}(\Omega)$,

$$
\int_{\Omega}|\nabla u|^{2} \mathrm{~d} x \geq \frac{(N-2)^{2}}{4} \int_{\Omega} \frac{u^{2}}{|x|^{2}} \mathrm{~d} x+z_{0}^{2}\left(\frac{\omega_{N}}{|\Omega|}\right)^{\frac{2}{N}} \int_{\Omega} u^{2} \mathrm{~d} x,
$$

where $\omega_{N}$ and $|\Omega|$ denote the volume of the unit ball and $\Omega$, respectively, and $\mathrm{z}_{0}=$ $2.4048 \ldots$ denotes the first zero of the Bessel function $J_{0}(z)$. Inequality (1.2) is optimal in case $\Omega$ is a ball centered at zero. Triggered by the work of Brezis and Vázquez (1.2), several Hardy inequalities have been established in recent years. In particular, Adimurthi et al.([2]) proved that, for $u \in C_{0}^{\infty}(\Omega)$, there exists a constant $C_{n, k}$ such that

$$
\int_{\Omega}|\nabla u|^{2} \mathrm{~d} x \geq \frac{(N-2)^{2}}{4} \int_{\Omega} \frac{u^{2}}{|x|^{2}} \mathrm{~d} x+C_{n, k} \sum_{j=1}^{k} \int_{\Omega} \frac{u^{2}}{|x|^{2}}\left(\prod_{i=1}^{j} \log ^{(i)} \frac{\rho}{|x|}\right)^{-2} \mathrm{~d} x,
$$

where

$$
\rho=\left(\sup _{x \in \Omega}|x|\right)\left(e^{e^{e . e^{e(k-t i m e s)}}}\right)
$$

(c) 2011 Xiao; licensee Springer. This is an Open Access article distributed under the terms of the Creative Commons Attribution License (http://creativecommons.org/licenses/by/2.0), which permits unrestricted use, distribution, and reproduction in any medium, provided the original work is properly cited. 
$\log ^{(1)}()=.\log ($.$) and \log ^{(k)}()=.\log \left(\log ^{(k-1)}().\right)$ for $k \geq 2$. Filippas and Tertikas ([3]) proved that, for $u \in C_{0}^{\infty}(\Omega)$, there holds

$$
\int_{\Omega}|\nabla u|^{2} \geq \frac{(N-2)^{2}}{4} \int_{\Omega} \frac{u^{2}}{|x|^{2}}+\frac{1}{4} \sum_{k=1}^{\infty} \int_{\Omega} \frac{u^{2}}{|x|^{2}} X_{1}^{2}\left(\frac{|x|}{D}\right) \cdots X_{k}^{2}\left(\frac{|x|}{D}\right),
$$

where $D \geq \sup _{x \in \Omega}|x|$,

$$
X_{1}(s)=(1-\ln s)^{-1}, X_{k}(s)=X_{1}\left(X_{k-1}(t)\right)
$$

for $k \geq 2$ and $\frac{1}{4}$ is the best constant in (1.4) and is never achieved. More recently, Ghoussoub and Moradifam ([4]) give a necessary and sufficient condition on a radially symmetric potential $V(|x|)$ on $\Omega$ that makes it an admissible candidate for an improved Hardy inequality. It states that the following improved Hardy inequality holds for $u \in C_{0}^{\infty}\left(B_{\rho}\right)$, where $B_{\rho}=\left\{x \in \mathbb{R}^{n}:|x|<\rho\right\}$,

$$
\int_{\Omega}|\nabla u|^{2} \mathrm{~d} x \geq \frac{(N-2)^{2}}{4} \int_{\Omega} \frac{u^{2}}{|x|^{2}} \mathrm{~d} x+\int_{\Omega} \frac{u^{2}}{|x|^{2}} V(|x|) \mathrm{d} x
$$

if and only if the ordinary differential equation

$$
\gamma^{\prime \prime}(r)+\frac{\gamma^{\prime}(r)}{r}+V(r) y(r)=0
$$

has a positive solution on $(0, \rho]$. These include inequalities (1.2)-(1.4).

Motivated by the work of Ghoussoub and Moradifam ([4]), in this note, we shall prove similar improved Hardy inequality on the Heisenberg group $\mathbb{H}^{n}$. Recall that the Heisenberg group $\mathbb{H}^{n}$ is the Carnot group of step two whose group structure is given by

$$
(x, t) \circ\left(x^{\prime}, t^{\prime}\right)=\left(x+x^{\prime}, t+t^{\prime}+2 \sum_{j=1}^{n}\left(x_{2 j} x_{2 j-1}^{\prime}-x_{2 j-1} x_{2 j}^{\prime}\right)\right) .
$$

The vector fields

$$
\begin{gathered}
X_{2 j-1}=\frac{\partial}{\partial x_{2 j-1}}+2 x_{2 j} \frac{\partial}{\partial t}, \\
X_{2 j}=\frac{\partial}{\partial x_{2 j}}-2 x_{2 j-1} \frac{\partial}{\partial t^{\prime}}
\end{gathered}
$$

$(j=1, \ldots, n)$ are left invariant and generate the Lie algebra of $\mathbb{H}^{n}$. The horizontal gradient on $\uplus^{n}$ is the (2n) -dimensional vector given by

$$
\nabla_{\mathbb{H}}=\left(X_{1}, \ldots, X_{2 n}\right)=\nabla_{x}+2 \Lambda x \frac{\partial}{\partial t},
$$

where $\nabla_{x}=\left(\frac{\partial}{\partial x_{1}}, \ldots, \frac{\partial}{\partial x_{2 n}}\right), \Lambda$ is a skew symmetric and orthogonal matrix given by

$$
\Lambda=\operatorname{diag}\left(J_{1}, \ldots, J_{n}\right), \quad J_{1}=\cdots=J_{n}=\left(\begin{array}{cc}
0 & 1 \\
-1 & 0
\end{array}\right) .
$$

For more information about $\mathbb{H}^{n}$, we refer to [5-8]. To this end we have: 


\section{Theorem 1.1}

Let $B_{R}=\left\{x \in \mathbb{R}^{2 n}:|x|<R\right\}$ and $\Omega_{H}=B_{R} \times \mathbb{R} \in \mathbb{H}^{n}$. Let $V(|x|)$ be a radially symmetric decreasing nonnegative function on $B_{R}$. If the ordinary differential equation

$$
\gamma^{\prime \prime}(r)+\frac{y^{\prime}(r)}{r}+V(r) y(r)=0
$$

has a positive solution on $(0, R]$, then the following improved Hardy inequality holds for $u \in C_{0}^{\infty}\left(\Omega_{H}\right)$

$$
\int_{\Omega_{H}}\left|\nabla_{\mathbb{H}} u\right|^{2} \mathrm{~d} x \mathrm{~d} t \geq(n-1)^{2} \int_{\Omega_{H}} \frac{u^{2}}{|x|^{2}} \mathrm{~d} x \mathrm{~d} t+\int_{\Omega_{H}} \frac{u^{2}}{|x|^{2}} V(|x|) \mathrm{d} x \mathrm{~d} t
$$

and the constant $(n-1)^{2}$ in (1.6) is sharp in the sense of

$$
(n-1)^{2}=\inf _{u \in C_{0}^{\infty}\left(\Omega_{H}\right) \backslash\{0\}} \frac{\int_{\Omega_{H}}\left|\nabla_{\mathbb{H}} u\right|^{2} \mathrm{~d} x \mathrm{~d} t}{\int_{\Omega_{H}} \frac{u^{2}}{|x|^{2}} \mathrm{~d} x \mathrm{~d} t} .
$$

\section{Corollary 1.2}

There holds, for $u \in C_{0}^{\infty}\left(\Omega_{H}\right)$,

$$
\int_{\Omega_{H}}\left|\nabla_{\mathbb{H}} u\right|^{2} \geq(n-1)^{2} \int_{\Omega_{H}} \frac{u^{2}}{|x|^{2}}+\frac{1}{4} \sum_{j=1}^{k} \int_{\Omega_{H}} \frac{u^{2}}{|x|^{2}}\left(\prod_{i=1}^{j} \log (i) \frac{R}{|x|}\right)^{-2}
$$

and the constant $1 / 4$ is sharp in the sense of

$$
\frac{1}{4}=\inf _{u \in C_{0}^{\infty}\left(\Omega_{H}\right) \backslash\{0\}} \frac{\int_{\Omega_{H}}\left|\nabla_{H} u\right|^{2}-(n-1)^{2} \int_{\Omega_{H}} \frac{u^{2}}{|x|^{2}}-\frac{1}{4} \sum_{j=1}^{k-1} \int_{\Omega_{H}} \frac{u^{2}}{|x|^{2}}\left(\prod_{i=1}^{j} \log ^{(i)} \frac{R}{|x|}\right)^{-2}}{\int_{\Omega_{H}} \frac{u^{2}}{|x|^{2}}\left(\prod_{i=1}^{k} \log ^{(i)} \frac{R}{|x|}\right)^{-2}} .
$$

\section{Corollary 1.3}

There holds, for $u \in C_{0}^{\infty}\left(\Omega_{H}\right)$ and $D \geq R$,

$$
\int_{\Omega_{H}}\left|\nabla_{\mathbb{H}} u\right|^{2} \geq(n-1)^{2} \int_{\Omega_{H}} \frac{u^{2}}{|x|^{2}}+\frac{1}{4} \sum_{k=1}^{\infty} \int_{\Omega_{H}} \frac{u^{2}}{|x|^{2}} X_{1}^{2}\left(\frac{|x|}{D}\right) \cdots X_{k}^{2}\left(\frac{|x|}{D}\right),
$$

and the constant $1 / 4$ is sharp in the sense of

$$
\frac{1}{4}=\inf _{u \in C_{0}^{\infty}\left(\Omega_{H}\right) \backslash\{0\}} \frac{\int_{\Omega_{H}}\left|\nabla_{H} u\right|^{2}-(n-1)^{2} \int_{\Omega_{H}} \frac{u^{2}}{|x|^{2}}-\frac{1}{4} \sum_{j=1}^{k-1} \int_{\Omega_{H}} \frac{u^{2}}{|x|^{2}} X_{1}^{2}\left(\frac{|x|}{D}\right) \cdots X_{j}^{2}\left(\frac{|x|}{D}\right)}{\int_{\Omega_{H}} \frac{u^{2}}{|x|^{2}} X_{1}^{2}\left(\frac{|x|}{D}\right) \cdots X_{k}^{2}\left(\frac{|x|}{D}\right)} .
$$

\section{Proof}

To prove the main result, we first need the following preliminary result. 


\section{Lemma 2.1}

Let $B_{R}=\left\{x \in \mathbb{R}^{2 n}:|x|<R\right\}$ and $V(|x|)$ be a radially symmetric decreasing nonnegative function on $B_{R}$. If the ordinary differential equation

$$
y^{\prime \prime}(r)+\frac{y^{\prime}(r)}{r}+V(r) y(r)=0
$$

has a positive solution on $(0, R]$, then the following improved Hardy inequality holds for $f \in C_{0}^{\infty}\left(B_{R}\right)$,

$$
\int_{B_{R}}\left|\partial_{r} f\right|^{2} \mathrm{~d} x \geq(n-1)^{2} \int_{B_{R}} \frac{f^{2}}{|x|^{2}} \mathrm{~d} x+\int_{B_{R}} \frac{f^{2}}{|x|^{2}} V(|x|) \mathrm{d} x
$$

where $r=|x|$ and $\partial_{r}=\frac{\langle x, \nabla\rangle}{|x|}$ is the radial derivation.

Proof

Observe that if $f$ is radial, i.e., $f(x)=f(r)$, then $|\nabla f|=\left|\partial_{r} f\right|$. By inequality (1.5), inequality (2.1) holds.

Now let $f \in C_{0}^{\infty}\left(B_{R}\right)$. If we extend $f$ as zero outside $B_{R}$, we may consider $f \in C_{0}^{\infty}\left(\mathbb{R}^{2 n}\right)$. Decomposing $f$ into spherical harmonics we get (see e.g., [9])

$$
f=\sum_{k=0}^{\infty} f_{k}(r) \phi_{k}(\sigma)
$$

where $\varphi_{k}(\sigma)$ are the orthonormal eigenfunctions of the Laplace-Beltrami operator with responding eigenvalues

$$
c_{k}=k(N+k-2), \quad k \geq 0 .
$$

The functions $f_{k}(r)$ belong to $C_{0}^{\infty}\left(B_{R}\right)$, satisfying $f_{k}(r)=O\left(r^{k}\right)$ and $f_{k}^{\prime}(r)=O\left(r^{k-1}\right)$ as $r \rightarrow 0$. So

$$
\int_{B_{R}}\left|\partial_{r} f\right|^{2} \mathrm{~d} x=\sum_{k=0}^{\infty} \int_{B_{R}}\left|f_{k}^{\prime}\right|^{2} \mathrm{~d} x
$$

and

$$
(n-1)^{2} \int_{B_{R}} \frac{f^{2}}{|x|^{2}} \mathrm{~d} x+\int_{B_{R}} \frac{f^{2}}{|x|^{2}} V(|x|) \mathrm{d} x=\sum_{k=0}^{\infty}\left((n-1)^{2} \int_{B_{R}} \frac{f_{k}^{2}}{|x|^{2}} \mathrm{~d} x+\int_{B_{R}} \frac{f_{k}^{2}}{|x|^{2}} V(|x|) \mathrm{d} x\right) .
$$

Note that if $f$ is radial, then inequality (2.1) holds. We have, since $f_{k}(r) \in C_{0}^{\infty}\left(B_{R}\right)$,

$$
\int_{B_{R}}\left|f_{k}^{\prime}\right|^{2} \mathrm{~d} x \geq(n-1)^{2} \int_{B_{R}} \frac{f_{k}^{2}}{|x|^{2}} \mathrm{~d} x+\int_{B_{R}} \frac{f_{k}^{2}}{|x|^{2}} V(|x|) \mathrm{d} x .
$$


Therefore, by (2.2) and (2.3),

$$
\begin{aligned}
\int_{B_{R}}\left|\partial_{r} f\right|^{2} \mathrm{~d} x & =\sum_{k=0}^{\infty} \int_{B_{R}}\left|f^{\prime}\right|^{2} \mathrm{~d} x \\
& \geq \sum_{k=0}^{\infty}\left((n-1)^{2} \int_{B_{R}} \frac{f_{k}^{2}}{|x|^{2}} \mathrm{~d} x+\int_{B_{R}} \frac{f_{k}^{2}}{|x|^{2}} V(|x|) \mathrm{d} x\right) \\
& =(n-1)^{2} \int_{B_{R}} \frac{f^{2}}{|x|^{2}} \mathrm{~d} x+\int_{B_{R}} \frac{f^{2}}{|x|^{2}} V(|x|) \mathrm{d} x .
\end{aligned}
$$

This completes the proof of lemma 2.1.

\section{Proof of Theorem 1.1}

Recall that the horizontal gradient on $\uplus^{n}$ is the (2n)-dimensional vector given by

$$
\nabla_{\mathrm{H}}=\left(X_{1}, \ldots, X_{2 n}\right)=\nabla_{x}+2 \Lambda x \frac{\partial}{\partial t},
$$

where $\nabla_{x}=\left(\frac{\partial}{\partial x_{1}}, \ldots, \frac{\partial}{\partial x_{2 n}}\right), \Lambda$ is a skew symmetric and orthogonal matrix given by

$$
\Lambda=\operatorname{diag}\left(J_{1}, \ldots, J_{n}\right), \quad J_{1}=\cdots=J_{n}=\left(\begin{array}{cc}
0 & 1 \\
-1 & 0
\end{array}\right) .
$$

Therefore, for any $\phi \in C_{0}^{\infty}\left(\mathbb{H}^{n}\right)$,

$$
\begin{aligned}
\left\langle x, \nabla_{\mathbb{H}} \phi\right\rangle & =\left\langle x, \nabla_{x} \phi\right\rangle+2\langle x, \Lambda x\rangle \frac{\partial \phi}{\partial t} \\
& =\left\langle x, \nabla_{x} \phi\right\rangle .
\end{aligned}
$$

Here we use the fact $\langle x, \Lambda x\rangle=0$ since $\Lambda$ is a skew symmetric matrix.

Since $u \in C_{0}^{\infty}\left(\Omega_{H}\right)$, for every $t \in \mathbb{R}, u(\cdot, t) \in C_{0}^{\infty}\left(B_{R}\right)$. By Lemma 2.1,

$$
\int_{B_{R}}\left|\partial_{r} u\right|^{2} \mathrm{~d} x \geq(n-1)^{2} \int_{B_{R}} \frac{u^{2}}{|x|^{2}} \mathrm{~d} x+\int_{B_{R}} \frac{u^{2}}{|x|^{2}} V(|x|) \mathrm{d} x
$$

Integrating both sides of the inequality (2.5) with respect to $t$, we have,

$$
\int_{\Omega_{H}}\left|\partial_{r} u\right|^{2} \mathrm{~d} x \mathrm{~d} t \geq(n-1)^{2} \int_{\Omega_{H}} \frac{u^{2}}{|x|^{2}} \mathrm{~d} x \mathrm{~d} t+\int_{\Omega_{H}} \frac{u^{2}}{|x|^{2}} V(|x|) \mathrm{d} x \mathrm{~d} t
$$

By (2.4) and the pointwise Schwartz inequality, we have

$$
\left|\partial_{r} u\right|=\frac{\left|\left\langle x, \nabla_{x} u\right\rangle\right|}{|x|}=\frac{\left|\left\langle x, \nabla_{\mathbb{H}} u\right\rangle\right|}{|x|} \leq\left|\nabla_{H_{H}} u\right| .
$$

Therefore, we obtain, by (2.6)

$$
(n-1)^{2} \int_{\Omega_{H}} \frac{u^{2}}{|x|^{2}} \mathrm{~d} x \mathrm{~d} t+\int_{\Omega_{H}} \frac{u^{2}}{|x|^{2}} V(|x|) \mathrm{d} x \mathrm{~d} t \leq \int_{\Omega_{H}}\left|\nabla_{H} u\right|^{2} \mathrm{~d} x \mathrm{~d} t .
$$

To see the constant $(n-1)^{2}$ is sharp, we choose $u(x, t)=\varphi(|x|) w(t)$ with $\phi(|x|) \in C_{0}^{\infty}\left(B_{R}\right)$ and $w(t) \in C_{0}^{\infty}(\mathbb{R})$. Since $\varphi$ is radial, we have 


$$
\begin{aligned}
\left|\nabla_{\overparen{H}} u(x, t)\right|^{2} & =\left\langle w(t) \nabla_{x} \phi(|x|)+2 \phi(|x|) \Lambda x w \prime(t), w(t) \nabla_{x} \phi(|x|)+2 \phi(|x|) \Lambda x w \prime(t)\right\rangle \\
& =\left|\nabla_{x} \phi(|x|)\right|^{2} w^{2}(t)+4|\Lambda x|^{2} \phi^{2}\left(w^{\prime}(t)\right)^{2}+4\left\langle\nabla_{x} \phi(|x|), \Lambda x\right\rangle \phi(|x|) w^{\prime}(t) \\
& =\left|\nabla_{x} \phi(|x|)\right|^{2} w^{2}(t)+4|\Lambda x|^{2} \phi^{2}(w \prime(t))^{2}+4 \phi \prime(|x|)\left\langle\frac{x}{|x|}, \Lambda x\right\rangle \phi(|x|) w \prime(t) \\
& =\left|\nabla_{x} \phi(|x|)\right|^{2} w^{2}(t)+4|x|^{2} \phi^{2}(|x|)(w \prime(t))^{2} .
\end{aligned}
$$

Here we use the fact $|\Lambda x|=|x|$ since $\Lambda$ is a orthogonal matrix. Therefore,

$$
\begin{aligned}
\frac{\int_{\Omega_{H}}\left|\nabla_{H} u\right|^{2} \mathrm{~d} x \mathrm{~d} t}{\int_{\Omega_{H}} \frac{u^{2}}{|x|^{2}} \mathrm{~d} x \mathrm{~d} t} & =\frac{\int_{\Omega_{H}}\left|\nabla_{x} \phi(|x|)\right|^{2} w^{2}(t)}{\int_{\Omega_{H}} \frac{\phi(|x|)^{2} w(t)^{2}}{|x|^{2}}}+4 \frac{\int_{\Omega_{H}}|x|^{2} \phi^{2}(|x|)\left(w^{\prime}(t)\right)^{2}}{\int_{\Omega_{H}} \frac{\phi(|x|)^{2} w(t)^{2}}{|x|^{2}}} \\
& =\frac{\int_{B_{R}}\left|\nabla_{x} \phi(|x|)\right|^{2} \mathrm{~d} x \cdot \int_{-\infty}^{+\infty} w^{2}(t) \mathrm{d} t}{\int_{B_{R}} \frac{\phi(|x|)^{2}}{|x|^{2}} \mathrm{~d} x \cdot \int_{-\infty}^{+\infty} w^{2}(t) \mathrm{d} t}+4 \frac{\int_{B_{R}}|x|^{2} \phi^{2}(|x|) \mathrm{d} x \cdot \int_{-\infty}^{+\infty}\left(w^{\prime}(t)\right)^{2}}{\int_{B_{R}} \frac{\phi(|x|)^{2}}{|x|^{2}} \mathrm{~d} x \cdot \int_{-\infty}^{+\infty} w^{2}(t) \mathrm{d} t} \\
& =\frac{\int_{B_{R}}\left|\nabla_{x} \phi(|x|)\right|^{2} \mathrm{~d} x}{\int_{B_{R}} \frac{\phi(|x|)^{2}}{|x|^{2}} \mathrm{~d} x}+4 \frac{\int_{B_{R}}|x|^{2} \phi^{2}(|x|) \mathrm{d} x}{\int_{B_{R}} \frac{\phi(|x|)^{2}}{|x|^{2}}} \cdot \frac{\int_{-\infty}^{+\infty}\left(w^{\prime}(t)\right)^{2}}{\int_{-\infty}^{+\infty} w^{2}(t) \mathrm{d} t}
\end{aligned}
$$

Since

$$
\inf _{w(t) \in C_{0}^{\infty}(\mathbb{R}) \backslash\{0\}} \frac{\int_{\mathbb{R}}\left|w^{\prime}(t)\right|^{2} \mathrm{~d} t}{\int_{\mathbb{R}}|w(t)|^{2} \mathrm{~d} t}=0,
$$

we obtain

$$
\inf _{u \in C_{0}^{\infty}\left(\Omega_{H}\right) \backslash\{0\}} \frac{\int_{\Omega_{H}}\left|\nabla_{\mathbb{H}} u\right|^{2} \mathrm{~d} x \mathrm{~d} t}{\int_{\Omega_{H}} \frac{u^{2}}{|x|^{2}} \mathrm{~d} x \mathrm{~d} t} \leq \inf _{\phi \in C_{0}^{\infty}\left(B_{R}\right) \backslash\{0\}} \frac{\int_{B_{R}}\left|\nabla_{x} \phi(|x|)\right|^{2} \mathrm{~d} x}{\int_{B_{R}} \frac{\phi(|x|)^{2}}{|x|^{2}} \mathrm{~d} x}=(n-1)^{2} .
$$

The proof of Theorem 1.1 is completed.

\section{Proof of Corollary 1.2}

By Theorem 1.1 and following [4], it is enough to show the constant $1 / 4$ is sharp. Choose $u(x, t)=\varphi(|x|) w(t)$ with $\phi(|x|) \in C_{0}^{\infty}\left(B_{R}\right)$ and $w(t) \in C_{0}^{\infty}(\mathbb{R})$. By the proof of Theorem 1.1,

$$
\left|\nabla_{\mathbb{H}} u(x, t)\right|^{2}=\left|\nabla_{x} \phi(|x|)\right|^{2} w^{2}(t)+4|x|^{2} \phi^{2}(|x|)\left(w^{\prime}(t)\right)^{2} .
$$

Therefore,

$$
\begin{aligned}
& \frac{\int_{\Omega_{H}}\left|\nabla_{H} u\right|^{2}-(n-1)^{2} \int_{\Omega_{H}} \frac{u^{2}}{|x|^{2}}-\frac{1}{4} \sum_{j=1}^{k-1} \int_{\Omega_{H}} \frac{u^{2}}{|x|^{2}}\left(\prod_{i=1}^{j} \log (i) \frac{R}{|x|}\right)^{-2}}{\int_{\Omega_{H}} \frac{u^{2}}{|x|^{2}}\left(\prod_{i=1}^{k} \log ^{(i)} \frac{R}{|x|}\right)^{-2}} \\
& =\frac{\int_{\Omega_{H}}\left|\nabla_{x} \phi(|x|)\right|^{2} w^{2}(t)-(n-1)^{2} \int_{\Omega_{H}} \frac{\phi^{2} w^{2}(t)}{|x|^{2}}-\frac{1}{4} \sum_{j=1}^{k-1} \int_{\Omega_{H}} \frac{\phi^{2} w^{2}(t)}{|x|^{2}}\left(\prod_{i=1}^{j} \log ^{(i)} \frac{R}{|x|}\right)^{-2}}{\int_{\Omega_{H}} \frac{\phi^{2} w^{2}(t)}{|x|^{2}}\left(\prod_{i=1}^{k} \log ^{(i)} \frac{R}{|x|}\right)^{-2}} \\
& =\frac{\int_{B_{R}}\left|\nabla_{x} \phi(|x|)\right|^{2}-(n-1)^{2} \int_{B_{R}} \frac{\phi^{2}}{|x|^{2}}-\frac{1}{4} \sum_{j=1}^{k-1} \int_{B_{R}} \frac{\phi^{2}}{|x|^{2}}\left(\prod_{i=1}^{j}|x|^{2} \phi^{2}(|x|)\left(w^{\prime}(t)\right)^{2}\right.}{\int_{\Omega_{H}} \frac{\phi^{2} w^{2}(t)}{|x|^{2}}\left(\prod_{i=1}^{k} \log ^{(i)} \frac{R}{|x|}\right)^{-2}} \\
& \int_{B_{R}} \frac{\phi^{2}}{|x|^{2}}\left(\prod_{i=1}^{k} \log ^{(i)} \frac{R}{|x|}\right)^{-2}
\end{aligned}
$$


Since

$$
\inf _{w(t) \in C_{0}^{\infty}(\mathbb{R}) \backslash\{0\}} \frac{\int_{\mathbb{R}}\left|w^{\prime}(t)\right|^{2} \mathrm{~d} t}{\int_{\mathbb{R}}|w(t)|^{2} \mathrm{~d} t}=0,
$$

we have

$$
\begin{aligned}
& \inf _{u \in C_{0}^{\infty}\left(\Omega_{H}\right) \backslash\{0\}} \frac{\int_{\Omega_{H}}\left|\nabla_{H} u\right|^{2}-(n-1)^{2} \int_{\Omega_{H}} \frac{u^{2}}{|x|^{2}}-\frac{1}{4} \sum_{j=1}^{k-1} \int_{\Omega_{H}} \frac{u^{2}}{|x|^{2}}\left(\prod_{i=1}^{j} \log ^{(i)} \frac{R}{|x|}\right)^{-2}}{\int_{\Omega_{H}} \frac{u^{2}}{|x|^{2}}\left(\prod_{i=1}^{k} \log ^{(i)} \frac{R}{|x|}\right)^{-2}} \\
& \leq \inf _{\phi(|x|) \in C_{0}^{\infty}\left(B_{R}\right) \backslash\{0\}} \frac{\int_{B_{R}}\left|\nabla_{x} \phi(|x|)\right|^{2}-(n-1)^{2} \int_{B_{R}} \frac{\phi^{2}}{|x|^{2}}-\frac{1}{4} \sum_{j=1}^{k-1} \int_{B_{R}} \frac{\phi^{2}}{|x|^{2}}\left(\prod_{i=1}^{j} \log ^{(i)} \frac{R}{|x|}\right)^{-2}}{\int_{B_{R}} \frac{\phi^{2}}{|x|^{2}}\left(\prod_{i=1}^{k} \log ^{(i)} \frac{R}{|x|}\right)^{-2}} \\
& =\frac{1}{4} .
\end{aligned}
$$

Here we use the fact that the sharp constant in inequality (1.3) is $1 / 4$ (see [4]). This completes the proof of Corollary 1.2.

\section{Proof of Corollary 1.3}

The proof is similar to that of Corollary 1.2 and it is enough to show the constant $1 / 4$ is sharp. Choose $u(x, t)=\varphi(|x|) w(t)$ with $\phi(|x|) \in C_{0}^{\infty}\left(B_{R}\right)$ and $w(t) \in C_{0}^{\infty}(\mathbb{R})$. Then

$$
\left|\nabla_{\mathbb{H}} u(x, t)\right|^{2}=\left|\nabla_{x} \phi(|x|)\right|^{2} w^{2}(t)+4|x|^{2} \phi^{2}(|x|)\left(w^{\prime}(t)\right)^{2} .
$$

Therefore,

$$
\begin{aligned}
& \frac{\int_{\Omega_{H}}\left|\nabla_{H} u\right|^{2}-(n-1)^{2} \int_{\Omega_{H}} \frac{u^{2}}{|x|^{2}}-\frac{1}{4} \sum_{j=1}^{k-1} \int_{\Omega_{H}} \frac{u^{2}}{|x|^{2}} X_{1}^{2}\left(\frac{|x|}{D}\right) \cdots X_{j}^{2}\left(\frac{|x|}{D}\right)}{\int_{\Omega_{H}} \frac{u^{2}}{|x|^{2}} X_{1}^{2}\left(\frac{|x|}{D}\right) \cdots X_{k}^{2}\left(\frac{|x|}{D}\right)} \\
= & \frac{\int_{\Omega_{H}}\left|\nabla_{x} \phi(|x|)\right|^{2} w^{2}(t)-(n-1)^{2} \int_{\Omega_{H}} \frac{\phi^{2} w^{2}(t)}{|x|^{2}}-\frac{1}{4} \sum_{j=1}^{k-1} \int_{\Omega_{H}} \frac{\phi^{2} w^{2}(t)}{|x|^{2}} X_{1}^{2}\left(\frac{|x|}{D}\right) \cdots X_{j}^{2}\left(\frac{|x|}{D}\right)}{\int_{\Omega_{H}} \frac{\phi^{2} w^{2}(t)}{|x|^{2}} X_{1}^{2}\left(\frac{|x|}{D}\right) \cdots X_{j}^{2}\left(\frac{|x|}{D}\right)} \\
= & +4 \frac{\int_{\Omega_{H}}|x|^{2} \phi^{2}(|x|)\left(w^{\prime}(t)\right)^{2}}{\int_{\Omega_{H}} \frac{\phi^{2} w^{2}(t)}{|x|^{2}} X_{1}^{2}\left(\frac{|x|}{D}\right) \cdots X_{j}^{2}\left(\frac{|x|}{D}\right)} \\
& +4 \frac{\int_{B_{R}}\left|\nabla_{x} \phi(|x|)\right|^{2}-(n-1)^{2} \int_{B_{R}} \frac{\phi^{2}}{|x|^{2}}-\frac{1}{4} \sum_{j=1}^{k-1} \int_{B_{R}} \frac{\phi^{2}}{|x|^{2}} X_{1}^{2}\left(\frac{|x|}{D}\right) \cdots X_{j}^{2}\left(\frac{|x|}{D}\right)}{|x|^{2}} X_{1}^{2}\left(\frac{|x|}{D}\right) \cdots X_{j}^{2}\left(\frac{|x|}{D}\right) \\
\int_{B_{R}} \frac{\phi^{2}}{|x|^{2}} X_{1}^{2}\left(\frac{|x|}{D}\right) \cdots X_{j}^{2}\left(\frac{|x|}{D}\right) & \frac{\int_{-\infty}^{+\infty}\left(w^{\prime}(t)\right)^{2}}{\int_{-\infty}^{+\infty} w^{2}(t) \mathrm{d} t} .
\end{aligned}
$$

Thus

$$
\begin{aligned}
& \inf _{u \in C_{0}^{\infty}\left(\Omega_{H}\right) \backslash\{0\}} \frac{\int_{\Omega_{H}}\left|\nabla_{H} u\right|^{2}-(n-1)^{2} \int_{\Omega_{H}} \frac{u^{2}}{|x|^{2}}-\frac{1}{4} \sum_{j=1}^{k-1} \int_{\Omega_{H}} \frac{u^{2}}{|x|^{2}} X_{1}^{2}\left(\frac{|x|}{D}\right) \cdots X_{j}^{2}\left(\frac{|x|}{D}\right)}{\int_{\Omega_{H}} \frac{u^{2}}{|x|^{2}} X_{1}^{2}\left(\frac{|x|}{D}\right) \cdots X_{k}^{2}\left(\frac{|x|}{D}\right)} \\
& \leq \inf _{\phi(|x|) \in C_{0}^{\infty}\left(B_{R}\right) \backslash\{0\}} \frac{\int_{B_{R}}\left|\nabla_{x} \phi(|x|)\right|^{2}-(n-1)^{2} \int_{B_{R}} \frac{\phi^{2}}{|x|^{2}}-\frac{1}{4} \sum_{j=1}^{k-1} \int_{B_{R}} \frac{\phi^{2}}{|x|^{2}}\left(\frac{|x|}{D}\right) \cdots X_{j}^{2}\left(\frac{|x|}{D}\right)}{\int_{B_{R}} \frac{\phi^{2}}{|x|^{2}}\left(\frac{|x|}{D}\right) \cdots X_{j}^{2}\left(\frac{|x|}{D}\right)} \\
& =\frac{1}{4} .
\end{aligned}
$$


since

$$
\inf _{w(t) \in C_{0}^{\infty}(\mathbb{R}) \backslash\{0\}} \frac{\int_{\mathbb{R}}\left|w^{\prime}(t)\right|^{2} \mathrm{~d} t}{\int_{\mathbb{R}}|w(t)|^{2} \mathrm{~d} t}=0 .
$$

This completes the proof of Corollary 1.3 .

\section{Acknowledgements}

The authors thanks the referee for his/her careful reading and very useful comments which improved the final version of this paper.

\section{Authors' contributions}

YX designed and performed all the steps of proof in this research and also wrote the paper. All authors read and approved the final manuscript.

\section{Competing interests}

The author declares that they have no competing interests.

Received: 2 April 2011 Accepted: 25 August 2011 Published: 25 August 2011

\section{References}

1. Brezis, H, Vázquez, JL: Blowup solutions of some nonlinear elliptic problems. Rev Mat Univ Comp Madrid. 10, 443-469 (1997)

2. Adimurthi, N, Chaudhuri, N, Ramaswamy, N: An improved Hardy Sobolev inequality and its applications. Proc Amer Math Soc. 130, 489-505 (2002). doi:10.1090/50002-9939-01-06132-9

3. Filippas, S, Tertikas, A: Optimizing improved Hardy inequalities. J Funct Anal. 192(1), 186-233 (2002). doi:10.1006/ jfan.2001.3900

4. Ghoussoub, N, Moradifam, A: On the best possible remaining term in the improved Hardy inequality. Proc Nat Acad Sci. 105(37), 13746-13751 (2008). doi:10.1073/pnas.0803703105

5. Garofalo, N, Lanconelli, E: Frequency functions on the Heisenberg group, the uncertainty principle and unique continuation. Ann Inst Fourier(Grenoble). 40, 313-356 (1990)

6. Luan, J, Yang, Q: A Hardy type inequality in the half-space on $\mathbb{R}^{n}$ and Heisenberg group. J Math Anal Appl. 347, 645-651 (2008). doi:10.1016/j.jmaa.2008.06.048

7. Niu, P, Zhang, H, Wang, Y: Hardy type and Rellich type inequalities on the Heisenberg group. Proc Amer Math Soc. 129 3623-3630 (2001). doi:10.1090/50002-9939-01-06011-7

8. Yang, Q: Best constants in the Hardy-Rellich type inequalities on the Heisenberg group. J Math Anal Appl. 342, 423-431 (2008). doi:10.1016/j.jmaa.2007.12.014

9. Tertikas, A, Zographopoulos, NB: Best constants in the Hardy-Rellich inequalities and related improvements. Adv Math. 209, 407-459 (2007). doi:10.1016/j.aim.2006.05.011

doi:10.1186/1029-242X-2011-38

Cite this article as: Xiao: An improved Hardy type inequality on Heisenberg group. Journal of Inequalities and Applications 2011 2011:38.

\section{Submit your manuscript to a SpringerOpen ${ }^{\circ}$ journal and benefit from:}

- Convenient online submission

- Rigorous peer review

- Immediate publication on acceptance

- Open access: articles freely available online

- High visibility within the field

- Retaining the copyright to your article

Submit your next manuscript at $\boldsymbol{s p r i n g e r o p e n . c o m ~}$ 